\title{
On the Second-Order Transition of a Rubber
}

\author{
E. A. DiMarzio*
}

(July 13, 1964)

\begin{abstract}
The view that the glass transition has a thermodynamic basis is extended to a crosslinked rubber. The elevation of the second-order transition temperature as a function of the number of cross-links is found to be $(T(X)-T(0)) / T(0)=K X / 1-K X$, where $T(X)$ is the transition temperature for a rubber with degree of cross-linking $X$. The constant $K$ is to first order independent of material. Also a relation involving no adjustable parameter is derived for the ratio of second-order transition temperatures to deformation. It is $T(\lambda) / T(1)=\exp \left(G\left(\Sigma \lambda_{i}{ }^{2}-3\right) / 2 \Delta C_{p} T_{0}\right)$ where $\Delta C_{p}$ is the value of the specific heat discontinuity per $\mathrm{cm}^{3}$ at the transition and $T_{0}$ is the temperature at which the modulus $G$ is measured. Available experimental evidence from five separate investigations agrees with these predictions.
\end{abstract}

\section{Introduction}

All noncrystalline polymers display what at first sight appears to be a second-order transition in the Ehrenfest sense [1]: ${ }^{1}$ the temperature and pressure derivatives of both volume and entropy are discontinuous along a line $T(P)$ although the volume and entropy themselves are continuous.

Many believe that this transition is basically a kinetic phenomenon because: (1) the location of the transition can be changed by changing the time scale of the experiment, faster measurements resulting in higher glass temperature, and (2) the measured relaxation times near the transition approach the time scale of the experiment. At temperatures below $T_{g}$ the relaxation time for certain molecular motions is larger than the time of observation and therefore there is a kinetic "freezing in" of the associated degrees of freedom.

Nonetheless we can state that these glass forming materials have equilibrium properties and ask what they are. One can answer this question experimentally by extrapolating the high temperature behavior of volume and entropy through the glass region. One finds that volumes and entropies smaller than the crystalline values are obtained at finite temperatures [2]. This unacceptable result can be avoided only if the curves level off before they cross the corresponding curves for the crystalline material. This behavior must be postulated even for infinite time scale experiments.

One can also ask what the theoretical predictions of equilibrium properties are. This question has been answered $[3,4]$ by means of a modified form of the Flory-Huggins lattice model which allows semiflexible chains to stiffen as the temperature is lowered [5]. This theory predicts a second-order transition at the finite temperature point at which the configurational entropy first becomes zero as the temperature of the system is lowered. The theory

\footnotetext{
*Present address: Polymer Physies Section, National Bureau of Standards, Washington, D.C., 20234; formerly with Bell Telephone Laboratories, Inc. Murray Hill, N.J.

1 Figures in brackets indicate the literature references at the end of this paper.
}

thus implies that packing difficulties cause the transition [3, 4]. This same lattice model also predicts correctly the existence and behavior of the various kinds of liquid crystals [6]. For these materials also, the extreme difficulty of packing in random arrangement causes the transition behavior $[6,7,8]$. The correct predictions in this case argue for the correctness of the predictions for glasses.

In addition the proposed statistical-mechanical theory correctly predicts the experimental data in those cases to which it has been applied [9]. Specifically, these are the variation of glass temperature with molecular weight and the variation with glass temperature of specific volume [4]. In addition the variation of glass temperature of a copolymer as a function of composition [10], and the variation of glass temperature with diluent [11] have been correctly predicted.

The purpose of this paper is to apply the theory to chemically cross-linked systems (rubbers) and to compare the predictions of the theory with the small amount of available experimental data.

\section{Relation Between the Transition Tem- perature and the number of Cross-Links}

The criterion of glass formation is that the temperature-dependent configurational entropy become zero. We therefore have for the equation which determines the second-order transition temperature,

$$
S_{c}=S_{0}+\Delta S_{1}=0
$$

where $S_{0}$ is the configurational entropy for an uncross-linked system and $\Delta S_{1}$ is the change in configurational entropy due to adding cross-links. Now one can immediately see that cross-linking raises the transition temperature because it decreases the configurational entropy over what it would be if there were no added cross-links. A cross-linked polymer on being cooled reaches the $S_{e}=0$ point at a higher temperature than the corresponding uncross-linked polymer. $S_{0}$ has been given previously as eq (20) of reference 4 . For our purposes we will 
use a simplified form obtained when the volume fraction approximation is used with appropriate substitutions from eq (11) and (24).

$$
\begin{gathered}
\frac{S_{0}}{k x N_{x}}=\ln \left(1+2 e^{-\frac{\Delta \epsilon}{k T}}\right)+f \frac{\Delta \epsilon}{k T}-1+\frac{T \Delta \alpha}{1+2 T \Delta \alpha} \\
f=2 e^{-\frac{\Delta \epsilon}{k T}} /\left(1+2 e^{-\frac{\Delta \epsilon}{k T}}\right) .
\end{gathered}
$$

The left-hand side is the dimensionless entropy per segment, $T$ is the temperature, $\Delta \epsilon$ is the "stiffness energy of the chain," $f$ is the fraction of bonds in upper wells, and $\Delta \alpha(T, P)$ the difference of thermal expansion coefficient for the material above the transition and the value below the transition. Equation 2 is valid for temperatures and pressures resulting in nonnegative values for $S_{0} / k x N_{x}$, but the pressure-temperature dependent value of $\Delta \alpha$ must be used. We will discuss actual numerical values of $\Delta \alpha$ later in the paper.

We can easily obtain $\Delta S_{1}$ by a simple modification of the Flory-Huggins counting scheme. Let us imagine that our system contains but one infinitely long polymer molecule before cross-linking. After cross-linking one can traverse each of the $N_{p}$ chains between cross-links once and only once by following along the full length of the original molecule. Let us then start at one end of the molecule which we will also assume to be tied to a cross-link and begin the Flory-Huggins counting process. The total number of ways to arrange a chain whose ends are tied down to given cells in the lattice is

$$
\begin{gathered}
\text { (F.H.) }\left[\frac{b^{3}}{\pi^{3 / 2}} \exp \left(-b^{2} r^{2}\right) d \tau\right], \\
b^{2}=(3 / 2) / n L^{2},
\end{gathered}
$$

where (F.H.) is that value which would obtain if one end were free, and the gaussian form gives the fraction of these conformations which are consistent with the end to end distance $\mathbf{r}$. The quantity $L$ is not the segment length $d$, but is a multiple of it

$$
L=c d \text {, }
$$

and $n$ is the number of these effective lengths.

The element of volume $d \tau$ is necessary because the quantity multiplying it within the brackets is a density. For the time being we restrict the ends to be in a given cell, so that $d \tau$ becomes equal to the volume of a lattice site, $d^{3}$. For the second chain and each successive chain we have a term identical in form to expression 3 . It is evident that after we have laid down the whole molecule we will have for the total number of arrangements consistent with the location of each cross-link to specified cells

$$
e^{S_{c} / k}=e^{S_{0} / k} \prod_{i=1}^{N_{p}}\left[\frac{b_{i}^{3}}{\pi^{3 / 2}} \exp \left(-b_{i}^{2} r_{i}^{2}\right) d \tau\right] .
$$

In point of fact the junctions are not restricted to particular places on the lattice but can be in any lattice site with probabilities determined by gaussian distributions [12]. For this reason we must assign an effective volume $V\left(N_{p}\right)$ to each of the $N_{p i} / 2$ junctions and a corresponding number $V\left(N_{p}\right) / d^{3}$ of cells which it can occupy. This gives for $\Delta S_{1}$

$$
e^{\Delta S_{1} / k}=\prod_{i=1}^{N_{p}}\left[\frac{b_{i}^{3}}{\pi^{3 / 2}} \exp \left(-b_{i}^{2} r_{i}^{2}\right) d \tau\left(\frac{V\left(N_{p}\right)}{d^{3}}\right)^{1 / 2}\right] .
$$

For the sake of simplicity we will assume that we can replace $n_{i}$ by $n$, the average number of effective lengths per chain. The effective volume $V\left(N_{p}\right)$ is a quantity whose linear dimensions are proportional to the breadth of the gaussian distribution. According: to James and Guth [12] this breadth is proportional to the square root of the average number of effective links between chains. Taking $V\left(N_{p}\right)$ inversely proportional to $b^{3}$,

$$
V\left(N_{p}\right)=A / b^{3}
$$

and assuming that the chains each have their average mean square lengths, we obtain

$$
\frac{\Delta S_{1}}{k}=-\frac{3}{2} N_{p}+\frac{3}{4} N_{p} \ln \left[\left(\frac{d \tau A^{1 / 2}}{(\pi d)^{3 / 2}}\right)^{4 / 3} / \frac{2}{3} n L^{2}\right] .
$$

Using (4) and the fact that the contour length is an invariant, i.e. $\left(x N_{x} / N_{p}\right) d=n L$, we get [13]

$$
\frac{\Delta S_{1}}{k}=-\frac{3}{2} N_{p}-\frac{3}{4} N_{p} \ln c+\frac{3}{4} N_{p} \ln \left(A^{\prime} X\right)
$$

where $X$, the cross-link density, is the number of moles of chains per mole of segments $\left(X=N_{p} / x N_{x}\right)$. Now, $c$ is the same value for all materials at the glass temperature. This is due to the fact that the number of segments in an effective link is a function of the stiffness of the chain, and at their glass temperatures all chains have the same [14] relative stiffness. This value of $c$ is obviously equal to the average number of segments between flexes.

$$
c=\frac{1}{f}
$$

The pure number $A^{\prime}$ has a value which is independent of material since both $d \tau$ and $V\left(N_{p}\right)$ were given in units of $d^{3}$ which has disappeared from the equation. We therefore have as our eq (11) relating secondorder transition temperature to degree of crosslinking [15].

$$
\begin{array}{r}
\frac{S_{c}}{k x N_{x}}=\ln \left(1+2 e^{-\Delta \epsilon / k T}\right)+f \Delta \epsilon / k T-1+\frac{T \Delta \alpha}{1+2 T \Delta \alpha} \\
-\frac{3}{2} X+\frac{3}{4} X \ln f+\frac{3}{4} X \ln \left(A^{\prime} X\right)=0 .
\end{array}
$$


The symbol $T$ will be used for glass temperature as well as temperature. No confusion should arise. As it stands eq (11) is presumably valid for all crosslinked polymers. In order to estimate the transition temperature three parameters specific to the material must be known: the number of moles of chains per mole of segments $X$, the stiffness energy $\Delta \epsilon$, and $\Delta \alpha$. As noted above, $A^{\prime}$ should not depend on material.

Equation 11 includes the effect of pressure, both internal due to cross-links [12], and external. Limiting ourselves to zero external pressure, we can write as an approximation

$$
T(X) \Delta \alpha(X)=T(0) \Delta \alpha(0) .
$$

In words, the glass temperature times $\Delta \alpha$ is a constant independent of amount of cross-linking. Equation (12) is first of all an experimental fact for natural rubber-sulfur vulcanizates [16]. Secondly, even if one were to assume $\Delta \alpha(X)$ a constant then the fourth term of eq (11) would vary with $T$ about $1 / 5$ as fast as the sum of the first terms, so that even for this extreme case a good first approximation would be to assume $T \Delta \alpha$ constant. Third, it is easy to see from the theory that as $T(X)$ rises due to cross-linking $\Delta \alpha(X)$ must decrease [17]. This phenomenon occurs because of the internal pressure which is built up as the cross-linking density increases. (See eq (3.8), of ref 12.) This sizable internal pressure squeezes out holes (or free volume) and results in a reduction of $\Delta \alpha$. We will therefore assume eq 12 to be true for all rubber systems.

We will approximate the $X$ dependent part of eq 11 by a straight line [15]. In view of the limited amount of experimental data, this is a perfectly adequate approximation, particularly since $X$ (which is the reciprocal of the number of segments per chain) is usually very small in the experiments.

$N_{p}$ is really equal to the number of effective chains formed when $N_{c}$ chemical chains are made minus the number of effective chains that exist (due to entanglements) when no chemical chains are made before cross-linking. If one accepts the data of Schaefgen and Flory [18] as typical for all polymers then $N_{p}=N_{c}$ to good approximation.

The net results of these developments is that we can replace eq (11) by a simpler relation of wide validity.

$$
\ln \left(1+2 e^{-\Delta \epsilon / k T}\right)+f \frac{\Delta \epsilon}{k T}-D+K_{1} X=0 .
$$

Here $K_{1}$ is independent of material and we have absorbed $T(0) \Delta \alpha(0)$ in $D$. The sum of the first two terms in eq 13 is to good approximation linear in $\Delta \epsilon / k T$. Using this fact one can easily express $\Delta \epsilon / k$ in terms of the glass temperature $T(0)$ of the uncrossed-linked material to get from eq (13)

$$
\frac{T(X)-T(0)}{T(0)}=\frac{K_{2} X}{1-K_{2} X}
$$

where $K_{2}$ is a pure number whose value is to first order independent of material.

There exists in the literature experimental data for variation of glass temperature as a function of number of cross-links for three polymer systems. They are: natural rubber [19], styrene-divinyl benzene copolymers $[20,21]$ (P.S.), and methyl methacrylate-ethylene glycol dimethacrylate copolymers [22] (P.M.M.). Since the experimental data are given in terms of number of cross-links per gram, we rewrite eq (14) as

$$
\frac{T(\chi)-T(0)}{T(0)}=\frac{K M \chi / \gamma}{1-K M \chi / \gamma}
$$

where $\chi$ is the number of cross-links per gram, $M$ is the molecular weight of a residue, and $\gamma$ is the number of flexible bonds (basic units) per residue. Of course $K$ is again independent of material. Perhaps $\gamma$ needs careful definition. It is defined as the number of rotatable bonds per residue which on rotating result in a change in the shape of the molecule. These flexible bonds can occur on side groups as well as in the backbone of the chain. Thus in polymethylmethacrylate $\gamma=4$ and $M / \gamma=25$. For natural rubber $\gamma$ is 3 since the repeat unit carries a double bond and $M / \gamma=22.7$. For polystyrene one can at first sight choose $\gamma$ equal to either 3 or 2 depending on whether or not rotation of the side group is held to cause a change in shape. Since steric hindrances exist it is reasonable to suppose there are two minima $180^{\circ}$ apart and that therefore $\gamma=2$, with $M / \gamma=52$. Also, this value agrees with the value for the basic chain unit of Wunderlich [23]. Using these values and the experimental values of $T$ and $\chi$ from references [19-22] we get for values of $K$,

Evaluation of the constant $K$

Natural rubber

P.S. rubber

P.M.M. rubber

$$
\begin{aligned}
& K=1.30 \times 10^{-23} \\
& K=1.20 \times 10^{-23} \\
& K=1.38 \times 10^{-23}
\end{aligned}
$$

These numbers agree remarkably well with each other and lend support to the contention that $K$ is independent of material.

On the basis of the assumption that all of the crosslinking agent actually cross-links, a linear relation rather than $14 \mathrm{a}$ was obtained by others [20, 22]. However, it is reasonable to suppose that at high density some cross-linking sites become immobilized in regions where there are no other such sites. It seems more reasonable to assume, in accordance with theory, that the modulus is proportional to $\chi$ and then evaluate the constant of proportionality by the chemical means at low cross-link density.

Now Martin and Mandelkern [16] have made a careful study of unaccelerated natural rubber-sulfur vulcanizates, and have measured the glass temperature as a function of bound sulfur content. In order for us to make a comparison we must know the relation between number of cross-links and percent 
bound sulfur. Gee [24] has made measurements which lead to the relation

$$
\frac{10^{4}}{M_{c}}=0.2 s
$$

where $s$ is percent bound sulfur and $M_{c}$ is molecular weight between cross-links, but his experiments extend only to a bound sulfur content of 7 percent. We will assume that this proportionality holds for larger amounts of bound sulfur [25]. In figure (1) we have plotted the data of Martin and Mandelkern and also fit the curve to this data. It is seen that the agreement is good out to a bound sulfur content of 20 percent. The equation used to fit this data was,

$$
\frac{T(X)-T(0)}{T(0)}=\frac{0.085 \times 10^{4} / M_{c}}{1-0.085 \times 10^{4} / M_{c}},
$$

where we have made use of eq (15). Thus as a function of the number of cross-links the data varies as per eq (14) if we assume that modulus is proportional to the number of chains.

The molecular weight between cross-links is a very difficult quantity to obtain. For this reason it is advantageous to express our relation in terms of variations of Young's Modulus $\left(G \alpha T N_{p}\right)$. We obtain

$$
\Delta T=T(X)-T(0)==K_{3} V_{s} M \Delta G / \gamma
$$

where $\Delta G$ is the difference in unstretched modulus between a cross-linked system and an uncross-linked system at their transition temperatures, $\Delta T$ is the difference between glass temperatures, and $V_{s}$ is the specific volume. Again, the coefficient $K_{3}$ should be independent of material.

In our derivation of eqs (17) and (14), we assumed that the cross-link was such that the two paired residues occupied adjacent sites on the lattice. If the cross-linking material were a long chain polymer we would need to count differently. One can see that the resulting equations are identical in form to eqs 17 and 14. All we need do is reinterpret $T(0)$ as the transition temperature of a linear copolymer formed by stringing together all of the chains into one long chain. $T(0)$ now of course varies as $X$ varies. A formula which adequately predicts the glass temperature of high molecular weight copolymers in terms of glass temperatures of the high molecular weight homopolymers has been given previously [10].

\section{Relation Between the Transition Tem- perature and the Degree of Strain in a Rubber}

The total configurational entropy for a strained rubber is

$$
S_{T}=S_{c}+\Delta S_{e}
$$

where $S_{c}$ has been discussed in section 2 and $\Delta S_{e}$, which is the change in configurational entropy as a function of strain, has been thoroughly and adequately discussed in the literature. Following Treloar [26] we use

$$
\Delta S_{e}=-\frac{1}{2} N_{p} k\left(\lambda_{1}^{2}+\lambda_{2}^{2}+\lambda_{3}^{2}-3\right)
$$

where $\lambda_{i}$ are the stretch ratios. We shall assume no volume changes $\left(\lambda_{1} \lambda_{2} \lambda_{3}=1\right)$. The isovolume assumption seems to be valid for extension ratios not exceeding five [26]. Thus as a practical measure it is a valid approximation for a dry rubber. However, for a swollen rubber this restriction would have to be relaxed and although this can be done it seems unlikely that the experimental consequences can be tested since, even in a dry rubber, measurements of glass temperature as a function of strain are exceedingly difficult.

We will use for $S_{c}$

$$
S_{c}=\int \frac{\Delta C_{p}}{T} d T
$$

which gives the temperature variation of $S_{c}$. Here $\Delta C_{p}$ is the total specific heat per $\mathrm{cm}^{3}$ of sample minus the extrapolated value for the glass.

The relation $S_{T}=0$ yields for the glass temperature $T(\lambda)$, if we assume $\Delta \dot{C}_{p}$ to be a constant,

$$
T(\lambda)=T(1) \exp \left(\frac{G}{2 \Delta C_{p} T_{0}}\left[\lambda_{1}^{2}+\lambda_{2}^{2}+\lambda_{3}^{2}-3\right]\right)
$$

where $G$ is the Modulus measured at temperature $T_{0}$. This is our end result.

One can ask why eq (20) was not used for $S_{0}$ in section 2 rather than eq (2). Had we done this we would have obtained

$$
T(X)=T(0) \exp \left(-\frac{K^{\prime} X}{\Delta C_{p}}\right)
$$

instead of eq (14). The first point is that $\Delta \mathrm{C}_{\gamma}$ at the glass temperature is a constant for all materials [27] when expressed per mole of flexible bonds so that both equations are universal relations. The second point is that as a function of temperature $\Delta C_{p}$ is not a constant but a decreasing function of $T$ [23, 28]. Over a wide range of temperature one jwould not expect eq (22) to hold since it was derived assuming $\Delta C_{p}$ constant. Thus our use of eq (2) is more accurate than assuming $\Delta C_{p}$ a constant. On the other hand if we are working in a small temperature range as is the case for eq [21] one can assume $\Delta C_{p}$ a constant.

Equation (21) contains no adjustable parameters and therefore provides a good test of the theory. All of the quantities that appear in eq (21) are independently measurable. Unfortunately the amount of data in the literature is meager and difficult to use for our purposes due to improper accounting of time effects. 


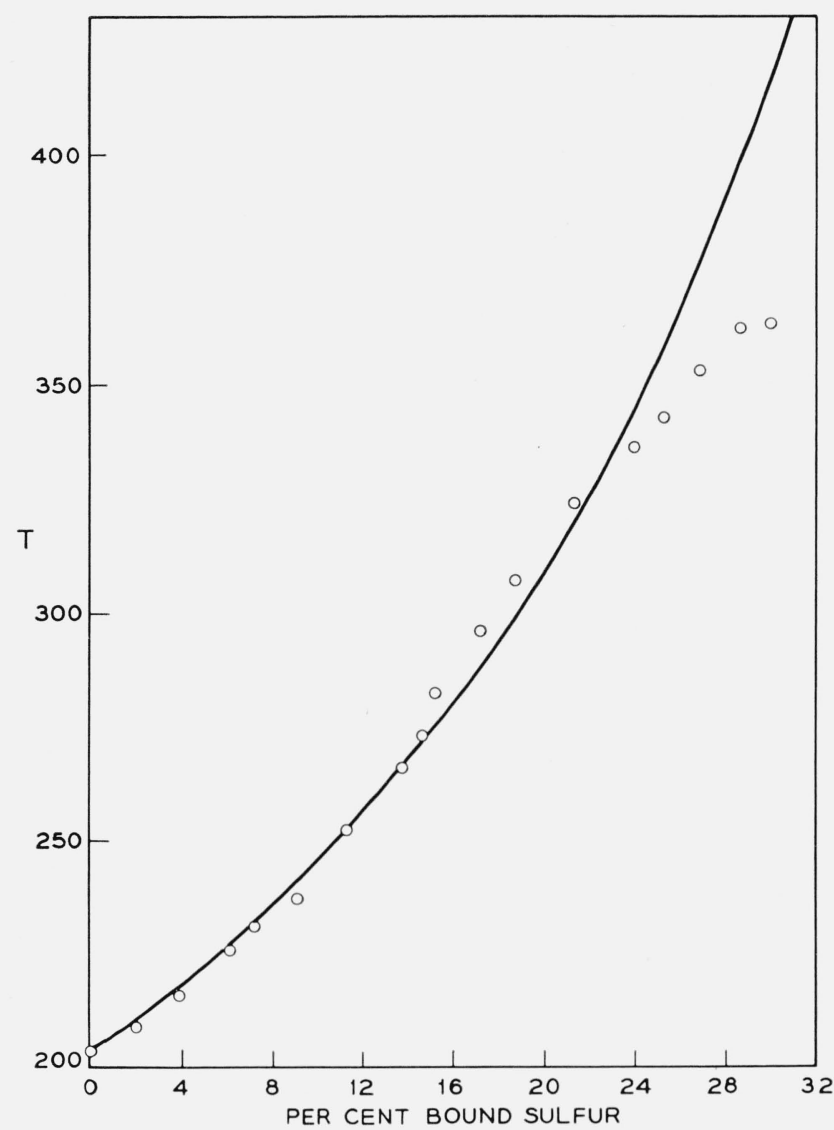

Figure 1. Variation of glass temperature $\mathrm{T}$ for natural rubber as a function of bound sulfur content.

The circles are experimental points of Martin and Mandelkern [16] and the line is theoretical.

Because of the nature of the quadratic form in the exponent of eq [21], and of the equation of constraint on the volume any deformation of the rubber from its unstretched state will result in an elevation of the transition temperature. The magnitude of this effect is shown in figure (2) for various values of the quantity $G / 2 \Delta C_{p} T_{\mathrm{o}}$ for the case of simple elongation. We have also calculated this quantity for materials used by Gee et al. [29] in some measurements of glass temperature as a function of elongation. The values are 0.0032 for their natural rubber sample, 0.0012 for G.R.S. and 0.0005 for Hycar. The specific heat values were obtained from Wunderlich [23] and the $G$ values were calculated from measurements made on figure (4) of reference (29). The values of $(T(\lambda)-T(0) / T(0)$ measured by Gee are also displayed. In view of the difficulties of the experiment any conclusions must be tentative. It seems however that agreement is satisfactory.

\section{Discussion}

The theory has made two very definite predictions. First, the elevation in transition temperature is proportional to the unstretched Young's Modulus of the rubber, and further the constant of proportional-



Figure 2. Variation of glass temperature as a function of elongation for various values of $\mathrm{G} / 2 \Delta \mathrm{C}_{p} \mathrm{~T}_{0}=\mathrm{F}$. Experimental points for natural rubber for which $F=0.0032$. E $\square$. .
Experimental points for G.R.S. for which $F=.0 .0012 . \quad \Delta$. Experimental point for Hyear for which $F=0.0005$

ity, $K_{3}$, is the same for all materials. Second, we have derived a relation with no adjustable parameter between the four independently measurable quantities: glass temperature, modulus, $\Delta C_{p}$ and $\lambda$. One hopes that more definitive experiments can be carried out in the near future. Further experimental validation of the relations in unison with the previously confirmed predictions would provide strong support for the theory.

However, were an alternative interpretation of the glass transition to predict the same relationships then these equations would carry much less weight. It therefore falls on us to examine the "Critical free volume theory" [30] and the more general "Order parameter theory" [31]. We shall find that the predictions are specific to the "Configurational entropy equals zero" theory.

\subsection{Free Volume Theory}

If one assumes that the glass forms when the free volume drops to a certain critical value one can 
easily derive as an approximation

$$
\Delta T=\frac{\Delta \beta}{3 \Delta \alpha} G
$$

where we have used the relation $P=G / 3$ of James and Guth [12] and eq (11.42) of reference [30]. Thus while our proportionality constant $K_{3}$ of eq (17) is to first order independent of material the free volume theory predicts by way of eq (25) a definite first order correlation with compressibility and expansion coefficients. Other free volume approaches are possible $[16,20]$ which result in equations different from eq (23).

In order to derive a free volume expression for the variation of transition temperature with amount of deformation in a rubber, one has simply to find a relation between free volume and degree of deformation. Since volume changes are to first order zero on stretching a rubber one would expect essentially no change in transition temperature with stretch ratios. In any event one would expect elevation of the transition temperature on compression and depression of transition temperature on elongation to the extent that there are any volume changes. Thus the direction of the effect for the case of simple elongation has opposite sign for the two theories in question.

\subsection{Order Parameter Theory}

The Order Parameter Theory as applied to glasses has been discussed by Davies and Jones [31].

Let us suppose that the Gibbs free energy $G$ of a polymer is dependent not only on $T$ and $P$ but also on an as yet unspecified number of quasi-thermodynamic variables $Z_{i}$. These variables will, if given enough time, adjust themselves so as to minimize the free energy; i.e.,

$$
\frac{\partial G}{\partial Z_{i}}=0
$$

Thus, at equilibrium one can use eq (24) to eliminate the $Z_{i}$ in terms of $T$ and $P$ so that thermodynamic variables are functions only of thermodynamic variables at equilibrium. For example the entropy is

$$
S=-\left.\frac{\partial G}{\partial T}\right|_{p}=-\left.\frac{\partial G}{\partial T}\right|_{p, Z_{i}}-\sum \frac{\partial G}{\partial Z_{i}} \frac{\partial Z_{i}}{\partial T}=S(T, P)
$$

One can say that at equilibrium the $Z_{i}$ are hidden variables.

For nonequilibrium situations eq (24) must be replaced by

$$
\frac{\partial G}{\partial Z_{i}}=\sum \eta_{i j} \dot{Z}_{j}
$$

where the $\eta_{i j}$ are generalized viscosities. Equation (26) assumes small deviations from equilibrium. Now because the $\eta_{i j}$ are temperature dependent there is a range of temperatures for which the $Z_{i}$ will reach their equilibrium values within the time scale of our experiments, and there is a range of (lower) temperatures for which they will not. Thus, we can say that the $Z_{i}$ became frozen in at sufficiently small temperatures. If one assumes this transition occurs over a small range of temperatures one has,

$$
\Delta S=-\left.\frac{\partial G}{\partial T}\right|_{p, Z_{i}}+\left.\frac{\partial G}{\partial T}\right|_{p, \mathbf{z}_{o i}}=0
$$

at the transition. That is to say, the entropy is continuous across the transition, but since the temperature derivatives are discontinuous we have a kinetically caused second-order transition.

If one uses $\lambda$ instead of $P$ in eq (27) one obtains by implicit differentiation of eq $(27)$ a relation between glass temperatures and strain.

$$
\frac{d T}{d \lambda}=-\frac{\frac{\partial \Delta S}{\partial \lambda}}{\frac{\partial \Delta S}{\partial T}}=\frac{T \frac{\partial \Delta f}{\partial T}}{\Delta C_{p}} .
$$

In applying eq (28) all measurements of $f$ must be made from surfaces generated by varying $T$ at constant $\lambda$. Similarly one can think of deoree of cross-linking as a thermodynamic variable and obtain,

$$
\frac{d T}{d X}=-\frac{T \frac{\partial \Delta S}{\partial X}}{\Delta C_{p}} .
$$

Equations (28) and (29) are differential analogs of eqs (14) and (21). They are valid even if the secondorder transition has a kinetic basis but they do presuppose that relaxation times are strongly temperature dependent.

These equations do not have the content of eqs (14) and (21) because they do not have a specified form for $\Delta S$. If we assume $\Delta S$ to be total configurational entropy then we do obtain (14) and (21). The above discussion shows that the "configurational entropy equals zero" theory is in some sense a variant of the order parameter theory if $\Delta S$ is itself considered to be an order parameter. For this reason it is proper to point out their differences and similarities.

Both theories assume quasi-thermodynamic variables. In the statistical thermodynamic theory used in this paper the number of empty lattice sites and the fraction of flexed bonds were such variables. At high temperatures both eqs (26) and (24) are applicable to both theories. As we lower the temperature the configurational entropy, $S_{c}$, becomes zero. At this temperature and for all lower temperatures we have as the equilibrium condition replacing (24),

$$
\frac{\partial\left(G-\mu S_{c}\right)}{\partial Z_{i}}=0, \quad S_{c}=0 .
$$

These equations lead to a second-order transition in the Ehrenfest sense. 
Since generalized forces (affinities) are measures of deviations from equilibrium we have in place of eq (26).

$$
\frac{\partial\left(G-\mu S_{c}\right)}{\partial Z_{i}}=\sum \eta_{i j} \dot{Z}_{j}
$$

Thus the predictions of both theories are the same above the transition and different below. The basis of the transition is thermodynamic in one and kinetic in the other.

It is a conclusion of eq (31) that the temperature derivatives of the $Z_{i}$ are discontinuous at the transition temperature. Hindsight tells us that this result was not unexpected. After all, for a first-order transition the time dependent quantities show discontinuities as we pass through the transition. In general the temperature dependence of time dependent quasi-thermodynamic variables shows a discontinuity of the same order as the transition. We hope to report on some of these aspects at a later date. However, even a cursory examination of the literature shows experimental behavior for relaxation quantities which can be interpreted as showing a change in slope as a function of temperature [32].

I thank F. H. Stillinger and W. P. Slichter of the Bell Telephone Laboratories for their constant encouragement and for some helpful sugeestions. I also thank E. Passaglia of NBS for helpful criticism of the manuscript.

\section{References and Notes}

[1] P. Ehrenfest, Leiden Comm. Suppl. 956 (1933).

[2] W. Kauzmann, Chem. Revs. 43, 219 (1948).

[3] J. H. Gibbs, J. Chem. Phys. 25, 185 (1956).

[4] J. H. Gibbs and E. A. Di Marzio, J. Chem. Phys. 28, 373 (1958).

[5] P. J. Flory, Proc. Roy. Soc. (London) A234, 60 (1956).

[6] E. A. DiMarzio, J. Chem. Phys. 35, 658 (1961).

[7] L. Onsager, Ann. N.Y. Acad. Sci. 51, 627 (1949).

[8] P. J. Flory, Proc. Roy. Soc. (London) A234, 73 (1956).

[9] In order to compare with experiment it is necessary tentatively to identify the true second-order transition as the actual glass temperature. The possibility that the thermodynamic transition occurs at some constant temperature difference below the observed transition has not been excluded. See, for example, the discussion section of reference [4].

[10] E. A. DiMarzio and J. H. Gibbs, J. Poly. Sci. 40, 121 (1959)

[11] E. A. DiMarzio and J. H. Gibbs, J. Poly. Sci. 1A, 1417 (1963).

[12] H. M. James and E. Guth, J. Chem. Phys. 11, 455 (1943).

[13] Equation (9) is identical in form to eq (15) of a paper by Flory and Rehner, J. Chem. Phys. 11, 512 (1943). The only difference is in the value for the argument of the logarithm. Our value is $c A^{\prime} X$, while theirs contain additionally several kinds of localization volumes which occur when one ties together structures formerly free. Thus one could use their equation if one could first explain away the $\Delta \tau^{\prime}$ s. Consider the Flory-Huggins formulas for the configurational entropy of two systems, one of $x$-mers and one of half as many $2 x$-mers. Neither of these formulas contain a $\Delta \tau$, yet if we were to calculate the entropy of the $2 x$-mers by pairing the $x$-mers we would have introduced a term $N_{2 x} \ln (\Delta \boldsymbol{r})$ in our expression for the entropy. Thus it becomes clear that the $\Delta \tau$ is nothing more than a measure of the cell size and that when we use a lattice model for our calculation of the configurational entropy of a rubber the $\Delta \tau$ should not appear.

[14] Chain stiffness is the main determinant of the transition. Intermolecular energies have as an effect which is normally small. The quantitative statement is obtained by equating the right hand side of eq 2 to zero.

[15] Were we to have used a "linear" model in which the number of junctions equaled the number of chains the $X$ dependent part of eq (11) would be strictly linear.

[16] G. M. Martin and L. Mandelkern, J. Res. NBS 62, 141 (1959) RP2944.

[17] The quantitative predictions are made by consideration of eq (11) and a simple extension of eq (11) of reference [4] with its resulting expression for $\Delta \alpha$ analogous to eq (24) of reference [4]. These equations plus the equation of James and Guth (eq (3.8) of ref. 12) relating internal pressure to degree of cross-linking are sufficient to determining both $\Delta \alpha(X)$ and $T(X)$ if $\Delta \alpha(0)$ and $T(0)$ are known.

[18] J. R. Schaefgen and P. J. Flory, J. Am. Chem. Soc. $\boldsymbol{7}$, $689(1950)$.

[19] P. Mason, J. Chem. Phys. 35, 1523 (1961)

[20] T. G. Fox and S. Loshaek, J. Poly. Sci. 15, 371 (1955).

[21] K. Ueberreiter and G. Kanig, J. Chem. Phys. 18, 399 (1950).

[22] S. Loshaek, J. Poly. Sci. 15, 391 (1955).

[23] B. Wunderlich, J. Phys. Chem. 64, 1052 (1960).

[24] G. Gee, J. Poly. Sci. 2, 451 (1947).

[25] The linear behavior of the specific volume versus percent bound sulfur curve provides a partial justification of this assumption (see fig. 3 of ref. 16). There are two reasons for this decrease over and above that in the rubber-sulfur mixtures; one is the formation of new chemical bonds, and the other is due to the fact that the large internal pressure compresses the system. According to James and Guth [12] this pressure is $1 / 3$ the modulus and therefore proportional to the number of individual chains. If we assume that the compressability is a constant then at small bound sulfur content the composite linear curve is due to two parts each of which is linear. Since the composite curve continues to be linear above 7 percent bound sulfur we can either assume that the two resolvent curves remain linear or that they are each nonlinear in such a way as to sum up to a linear curve. But the latter alternative would be fortuitous since the individual curves arise from unrelated causes.

[26] L. R. G. Treloar, The Physics of Rubber Elasticity, 2d eidtion, Oxford University Press, (1958).

[27] This has been observed by Wunderlich (ref. 23). The theory also predicts this result since to first order $\Delta C_{p}$ is a function only of $\Delta \epsilon / k T$ which is a constant at the glass temperature.

[28] G. T. Furukawa and M. L. Reilly, J. Res. NBS 56, 285 (1956) RP 2676.

[29] G. Gee, P. N. Hartley, J. B. M. Herbert and H. A. Lanceley, Polymer 1, 365 (1960).

[30] J. D. Ferry Viscoelastic Properties of Polymers (John Wiley \& Sons, Inc., New York, N.Y., 1961).

[31] R. O. Davies and G. O. Jones, Advances in Physics 2, 370 (1953).

[32] Examine for example figure 7 from: A. Kovacs, J. Poly. Sci. 30, 131 (1958).

(Paper 68A6-308) 